\title{
Ambipolar organic semiconductor blends for photovoltaic cells
}

\author{
Andreas Opitz, Markus Bronner, Julia Wagner, \\ Marcel Götzenbrugger, Wolfgang Brütting \\ Institute of Physics, University of Augsburg, 86135 Augsburg, Germany
}

\begin{abstract}
One material system of interest for photovoltaic cells is the combination of the $p$-conducting copper-phthalocyanine $(\mathrm{CuPc})$ and the $n$-conducting fullerene $\left(\mathrm{C}_{60}\right)$ as donor and acceptor materials, respectively. Therefore the transport properties for diodes containing neat and blended organic films are analysed in the space charge limited current regime. The charge carrier mobilities are found to decrease upon dilution of the respective conducting phase by the other species. Photovoltaic cells can be realised with bilayered or blended organic donor/acceptor films. The influence of both photo-active layer types on the electronic structure and the open circuit voltage is investigated. From photoelectron spectroscopy a higher open circuit voltage is predicted for bilayered solar cells. Due to mixing of the organic materials the intermolecular gap between the highest occupied molecular orbital of the donor and the lowest unoccupied molecular orbital of the acceptor is reduced. This prediction is proven true by photocurrent measurements.
\end{abstract}

\section{INTRODUCTION}

Organic donor and acceptor materials are promising candidates for photovoltaic cells. At the donor/acceptor interface in these cells the generated excitons dissociate into free charge carriers by an ultra-fast charge transfer in the sub-picosecond range. ${ }^{1,2}$ To create this donor/acceptor interface bilayered (heterojunction) ${ }^{3}$ and blended (bulk-heterojunction $)^{4,5}$ photovoltaic cells are employed (see figure 1a). In bilayered systems the dissociation interface is localised between the two layers, while the dissociation in the bulk-heterojunction cells occurs within the whole volume of the blended film (see figure 1a). Due to this mixture of donor and acceptor materials the photon-to-current conversion efficiency and the power conversion efficiency were found to increase. ${ }^{4-6}$ One molecular material system of interest for photovoltaic cells is the combination of copper-phthalocyanine $(\mathrm{CuPc})$ as $p$-conducting donor and fullerene $\left(\mathrm{C}_{60}\right)$ as $n$-conducting acceptor. ${ }^{6-10}$

Apart from solar cells, organic blends have also been applied in ambipolar field-effect transistors. ${ }^{11}$ The devices show an exponential decrease of the charge carrier mobilities upon reducing the concentration of the respective transport material (e.g. $\mathrm{CuPc}$ and $\left.\mathrm{C}_{60}\right) \cdot{ }^{12-14}$ This effect indicates that percolation or grain boundaries are the limiting factors for charge transport in the analysed blends of molecular organic semiconductors. The different charge carrier types are transported in the respective transport material and by mixing the hopping distance between the molecules or the grains increases. ${ }^{13}$

The electronic structure of blended molecular organic semiconductors ( $\mathrm{CuPc}$ and $\mathrm{C}_{60}$ on gold electrodes) was already analysed in dependence on the concentration. ${ }^{15,16}$ No indications for charge transfer or chemical reactions in the ground state were found. The ionisation potential of the highest occupied molecular orbital (HOMO) and the core levels of the two involved molecules are unchanged by mixing the materials. Assuming a constant energy gap and a constant electron affinity for the lowest unoccupied molecular orbital (LUMO) of both materials, independent of the mixing ratio, a reduced open circuit voltage in blended solar cells was predicted. ${ }^{15}$

In this contribution the analysis of charge carrier transport in diodes containing neat and blended films of $\mathrm{CuPc}$ and $\mathrm{C}_{60}$ will be reported. The dependence of the mobility on the mixing ratio should extend the knowledge from field-effect measurements. The electronic structure of blended and bilayered systems is analysed on a solar cell relevant electrode material (indium-tin-oxide, ITO) covered with a conducting polymer (polyethylenedioxythiophene-polystyrenesulfonate, PEDOT:PSS) and will be compared to bilayered and blended solar cells. Of particular interest will be the difference in the open circuit voltage between bilayered and blended solar cells.

Further author information: (Send correspondence to Andreas Opitz)

E-mail: Andreas.Opitz@physik.uni-augsburg.de

Photonics for Solar Energy Systems II, edited by Andreas Gombert

Proc. of SPIE Vol. 7002, 70020J, (2008) · 0277-786X/08/\$18 $\cdot$ doi: 10.1117/12.780998

Proc. of SPIE Vol. 7002 70020J-1 
(a) Bilayered system

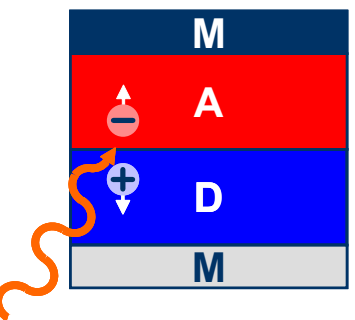

Blended system

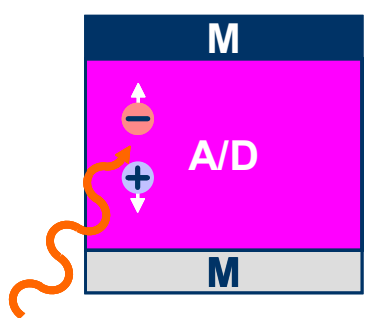

(b) Buckminster-Fullerene

$\mathrm{C}_{60}$

Copper-Phthalocyanine

$\mathrm{CuPc}$

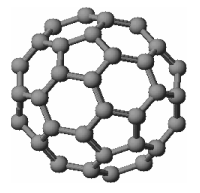

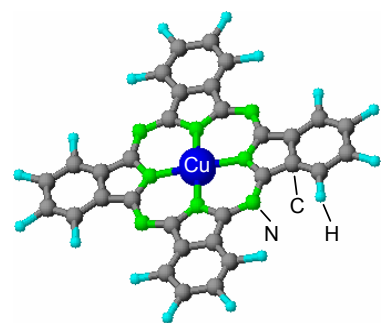

Figure 1. (a) Side view of the bilayered and blended solar cell, including metal electrodes (M), the donor (D) and the acceptor (A) molecules. Additionally the charge transport is sketched. (b) Chemical structures of the used organic semiconductors: copper-phthalocyanine $(\mathrm{CuPc})$ and fullerene $\left(\mathrm{C}_{60}\right)$.

\section{MATERIALS AND PREPARATION}

Copper-phthalocyanine $(\mathrm{CuPc}$, purchased from Sigma Aldrich and additionally purified by temperature gradient sublimation) and buckminster fullerene $\left(\mathrm{C}_{60}\right.$, purchased from Sigma Aldrich as sublimation grade) were used as organic semiconductors (OSC). The structural formulas are given in figure 1b. The organic semiconductor films were deposited by thermal evaporation from low-temperature effusion cells in a vacuum better than $1 \times 10^{-7} \mathrm{mbar}$. The thickness of the films was controlled via deposition monitors using quartz microbalances. For mixed films two independent monitors were used. The deposition rates were $0.35 \AA / \mathrm{s}$ for neat films and up to $1.4 \AA / \mathrm{s}$ for the material with the higher volume fraction in the mixtures.

The charge transport properties are analysed in both unipolar and ambipolar diodes. For the hole-only diode a bottom contact of indium-tin oxide (ITO) covered with $30 \mathrm{~nm}$ of the conducting polymer polyethylenedioxythiophene-polystyrenesulfonate (PEDOT:PSS, purchased from H.C. Starck as BAYTRON P) was used. The active organic layer was deposited on the PEDOT:PSS and after that a $40 \mathrm{~nm}$ thick N,N'-bis(3-methylphenyl)(1,1'-biphenyl)-4,4'-diamine (TPD) film to prevent electron injection from the $30 \mathrm{~nm}$ thick gold top electrode. TPD is known as electron blocking layer and can be neglected for the mobility analysis due to its high hole mobility. ${ }^{17}$ The electron-only diodes contain a $30 \mathrm{~nm}$ thick $\mathrm{Al}$ electrode at the bottom and a $30 \mathrm{~nm}$ thick $\mathrm{Al}$ electrode on the top of the organic film with a $0.5 \mathrm{~nm}$ thick interface doping layer of LiF. Using the ITO/PEDOT:PSS together with $\mathrm{LiF} / \mathrm{Al}$ electrodes ambipolar injection and charge transport occurs. The active organic layer is in all cases a $200 \mathrm{~nm}$ thick film of neat or blended materials with different mixing ratios. The active area is about $2 \times 2 \mathrm{~mm}^{2}$.

To analyse the current-voltage characteristics the measured curves were fitted by the model of trap-free space charge limited current (SCLC): ${ }^{18}$

$$
j_{\mathrm{SCLC}}=\frac{9}{8} \cdot \mu \cdot \varepsilon_{0} \varepsilon_{\mathrm{r}} \frac{V^{2}}{d^{3}}
$$

In this equation $V$ is the applied voltage reduced by the built-in voltage $\left(V_{\mathrm{Bi}}\right)$ which occurs due to different work functions of the electrodes, $d$ the thickness of the semiconducting film and $\varepsilon_{\mathrm{r}}$ the dielectric constant of the organic semiconductor. Additionally a Poole-Frenkel type field-dependent mobility ${ }^{19}$ is considered, which gives the current density as

$$
j_{\mathrm{SCLC}}=\frac{9}{8} \cdot \mu_{0} \cdot \varepsilon_{0} \varepsilon_{\mathrm{r}} \frac{V^{2}}{d^{3}} \cdot \exp \left[0.89 \cdot \gamma \cdot \sqrt{\frac{V}{d}}\right] .
$$

This dependence contains the zero-field mobility $\mu_{0}$ and the field activation parameter $\gamma$. The parameters $\mu_{0}, \gamma$ and $V_{\mathrm{Bi}}$ are determined by fitting the measurements in the higher voltage range. 
For the analysis by photoelectron spectroscopy a blended film of $25 \mathrm{~nm}$ thickness was evaporated on the PEDOT:PSS/ITO substrate. The mixing ratio of $\mathrm{CuPc}_{\mathrm{C}} \mathrm{C}_{60}=3: 1$ was determined by X-ray photoelectron spectroscopy. The bilayered structure consists of a $25 \mathrm{~nm} \mathrm{C}_{60}$ film deposited on a $25 \mathrm{~nm}$ thick film of CuPc, where the latter was again grown on a PEDOT:PSS covered ITO substrate. For comparison also a neat CuPc film with a thickness of $25 \mathrm{~nm}$ was analysed. The electronic properties of the films were characterised using X-ray and ultraviolet photoelectron spectroscopy (UPS) by employing monochromated Al K $\alpha$ radiation $(h \nu=1486.7 \mathrm{eV})$ to measure the core levels (not shown here) as well as ultraviolet radiation $\mathrm{He} \mathrm{I}(h \nu=21.2 \mathrm{eV})$ and $\mathrm{He} \mathrm{II}(h \nu=40.8 \mathrm{eV})$ for an analysis of the occupied states near the Fermi level.

The solar cells have a total organic film thickness of $80 \mathrm{~nm}$, comprising either a 1:1 mixture or a $40 \mathrm{~nm} \mathrm{C}_{60}$ film on top of a $40 \mathrm{~nm} \mathrm{CuPc}$ film (like sketched in figure 1a). As top electrode for electron extraction LiF/Al finishes the solar cells. Current-voltage characteristics of the solar cells were measured in darkness and under illumination. The intensity of the solar simulator (AM1.5 filters) was ranged up to $20 \mathrm{~mW} / \mathrm{cm}^{2}$, i.e. $0.2 \mathrm{suns}$.

\section{CHARGE CARRIER TRANSPORT}

The charge carrier transport is analysed in diodes allowing electron-only (device structure: $\mathrm{Al} / \mathrm{OSC} / \mathrm{LiF} / \mathrm{Al}$ ), hole-only (ITO/PEDOT:PSS/OSC/TPD/Au) or ambipolar (ITO/PEDOT:PSS/OSC/LiF/Al) transport. The

(a)

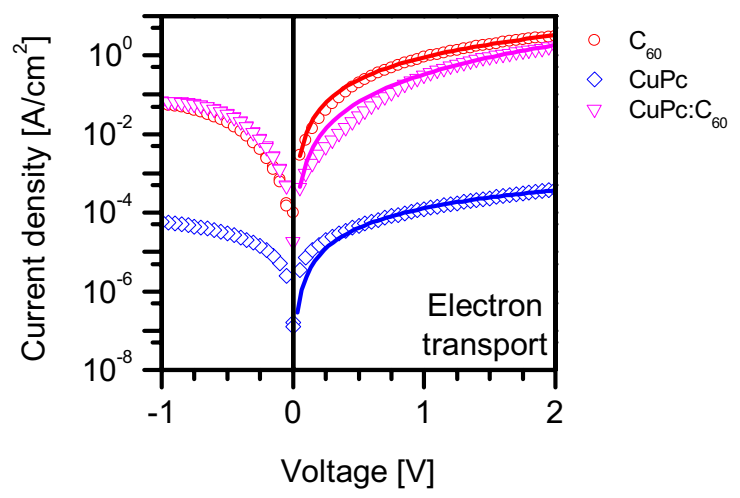

(c)

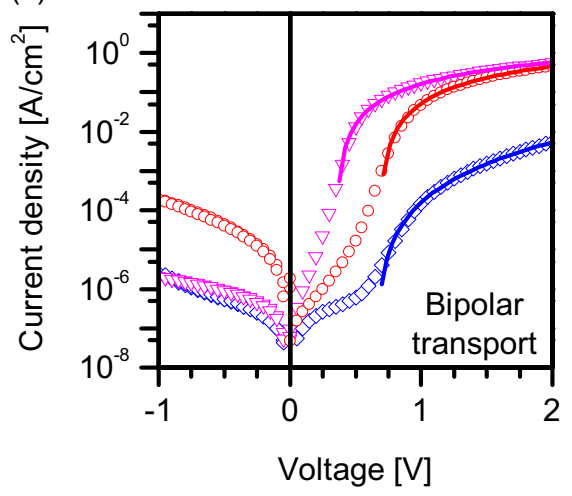

(b)

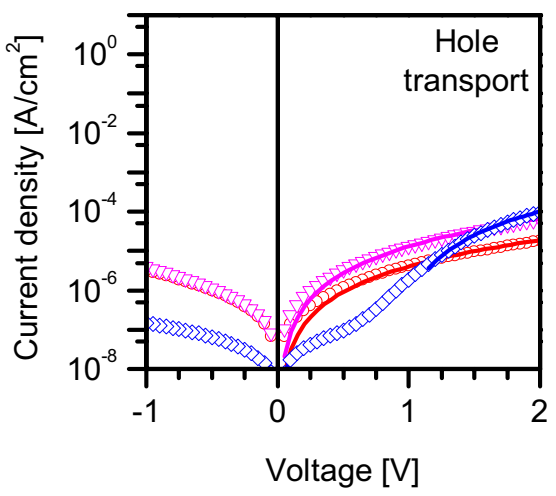

(d)

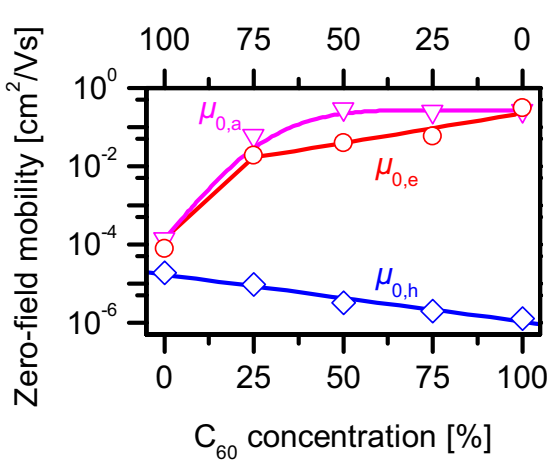

Figure 2. Dependence of the current density on the applied voltage for electron transport (a), hole transport (b) and ambipolar transport (c). The symbols are the measured values, the lines are fits using the SCLC model from equation 2. The mixing ratio of $\mathrm{CuPc}: \mathrm{C}_{60}$ in the blended film is about $1: 1$. The resulting zero-field mobilities $\left(\mu_{0}\right)$, including those of a 3:1 and a 1:3 blend, are shown in part (d). The lines are to guide the eyes. 
Table 1. Extracted parameters from the unipolar and ambipolar diode measurements. The zero-field mobility $\mu_{0}$ is given in $\mathrm{cm}^{2} / \mathrm{Vs}$ and the field activation parameter $\gamma$ in $(\mathrm{cm} / \mathrm{V})^{1 / 2}$.

\begin{tabular}{c|ccccc}
\hline & \multicolumn{5}{|c}{ Mixing ratio $-\mathrm{C}_{60} / \mathrm{CuPc}$} \\
SCLC parameters & $\mathrm{C}_{60}$ & $3: 1$ & $1: 1$ & $1: 3$ & $\mathrm{CuPc}$ \\
\hline$\mu_{0, \mathrm{e}}$ & $3.1 \times 10^{-1}$ & $5.7 \times 10^{-2}$ & $4.0 \times 10^{-5}$ & $1.9 \times 10^{-5}$ & $7.8 \times 10^{-5}$ \\
$\gamma_{\mathrm{e}}$ & - & $2.6 \times 10^{-4}$ & $3.9 \times 10^{-4}$ & $5.2 \times 10^{-5}$ & - \\
\hline$\mu_{0, \mathrm{~h}}$ & $1.3 \times 10^{-6}$ & $1.9 \times 10^{-6}$ & $3.3 \times 10^{-6}$ & $9.3 \times 10^{-6}$ & $1.9 \times 10^{-5}$ \\
$\gamma_{\mathrm{h}}$ & $1.9 \times 10^{-4}$ & - & $3.0 \times 10^{-4}$ & $2.2 \times 10^{-4}$ & $3.6 \times 10^{-4}$ \\
\hline$\mu_{0, \mathrm{a}}$ & $2.6 \times 10^{-1}$ & $2.5 \times 10^{-1}$ & $2.9 \times 10^{-1}$ & $6.0 \times 10^{-2}$ & $1.4 \times 10^{-4}$ \\
$\gamma_{\mathrm{a}}$ & $6.9 \times 10^{-4}$ & $5.7 \times 10^{-4}$ & $6.5 \times 10^{-4}$ & $4.0 \times 10^{-4}$ & - \\
\hline
\end{tabular}

measured dependence of the current density on the applied voltage is shown in figure 2a-c for both neat films and a blended film with a mixing ratio of 1:1. Additionally the 3:1 and the 1:3 mixing ratio was analysed, but not shown here. The current asymmetries between forward and reversed bias are related to the different injecting behaviours of the bottom and the top electrode, which is pronounced mostly for the ambipolar measurements. The curves are fitted using the described SCLC model including a mobility depending on the electric field. While the built-in voltage can be neglected for the unipolar transport (except the hole transport in CuPc due to the additional TPD layer), $V_{\mathrm{Bi}}$ is pronounced for all ambipolar measurements, originating from the difference of the electrode work functions. It is remarkable, that the built-in voltage is independent on the mixing ratio of the blended films and with a value of about $0.35 \mathrm{~V}$ much lower than the built-in voltage of the diodes containing neat films $\left(V_{\mathrm{Bi}}=0.67 \mathrm{eV}\right)$.

The determined zero-field mobility is shown in figure $2 \mathrm{~d}$ and collected together with the field activation parameter $\gamma$ in table 1 . The field activation parameter is low and sometimes even negligible. With the used electrode materials the transport of both charge carrier types is observed in $\mathrm{C}_{60}$ as well as CuPc. However, the unipolar mobilities depend strongly on the mixing ratio. The electron mobility decreases exponentially with decreasing $\mathrm{C}_{60}$ content and in the neat $\mathrm{CuPc}$ film a further reduction of the mobility occurs. From this it can be deduced, that the electron transport in the blends is carried by the $\mathrm{C}_{60}$ molecules only. By mixing the hopping distance is increased and as result the mobilities decrease exponentially. The strong mobility decrease between the mixed film with the highest $\mathrm{CuPc}$ content and the neat $\mathrm{CuPc}$ film is related to the much lower electron mobility in neat $\mathrm{CuPc}$. By contrast, the hole mobility changes over the whole concentration range uniformly, as the difference in the hole mobility between the two materials is much smaller. The same behavior, the exponentially reduced mobility with dilution, has already been observed in field-effect transistors using this material system. ${ }^{12,13}$ In contrast to the field-effect transistor measurements and results from the literature ${ }^{10}$ no balanced mobilities for electrons and holes were found in diodes. This is probably due to the high asymmetry between the hole mobility in $\mathrm{CuPc}$ and the electron mobility in $\mathrm{C}_{60}$.

As afore mentioned both molecular materials can transport electrons as well as holes in the used electrode configuration. Using an electron and a hole injecting electrode ambipolar transport occurs. The determined mobility for this ambipolar transport (shown also in figure 2d) is higher than the sum of the unipolar hole and unipolar electron mobility. This should be related to the ambipolar nature of both materials. Nevertheless the transport in the blended films is based mainly on the transport of electrons by the $\mathrm{C}_{60}$ molecules. Conductive paths of the spherical $\mathrm{C}_{60}$ molecules are also expected in the presence of the planar stacking $\mathrm{CuPc}$ molecules in the mixture. ${ }^{10,12,13}$

\section{ELECTRONIC STRUCTURE}

The occupied states near the Fermi level were analysed by UPS. The measured curves for the blended and the bilayered system are shown as solid lines in figure 3 . The features visible in the spectra are related to $\mathrm{CuPc}^{15,20}$ with the clearly separated $\mathrm{HOMO}$ peak. The $\mathrm{C}_{60} \mathrm{HOMO}$ has an about $1 \mathrm{eV}$ higher binding energy and lies in the 

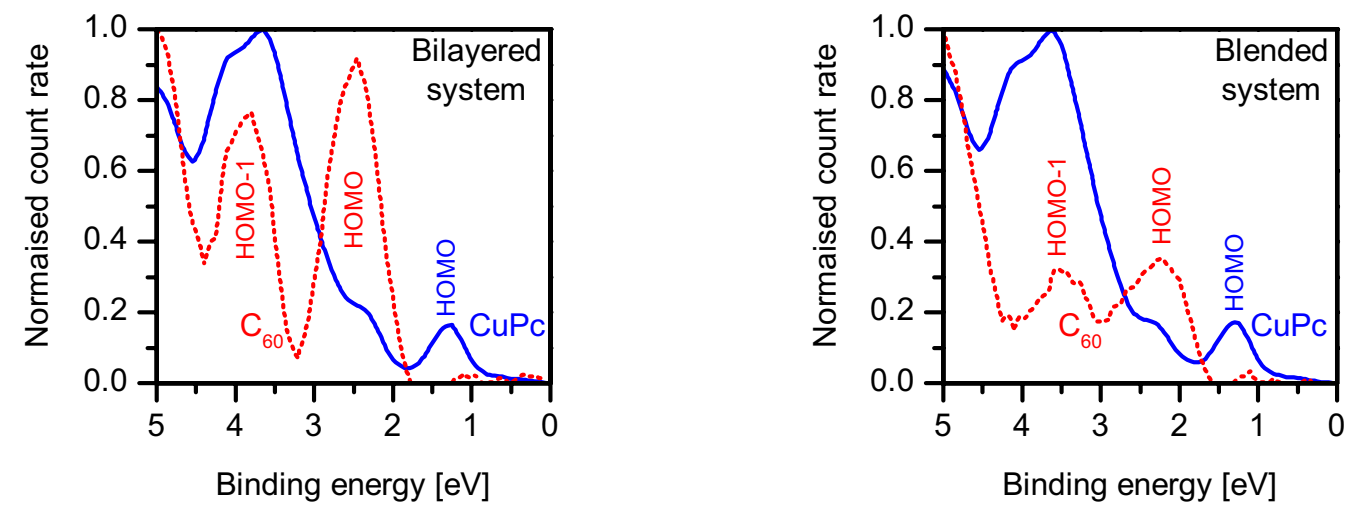

Figure 3. UPS spectra for the bilayered (left side) and the blended (right side) systems. The solid lines give the direct measurements where mainly the $\mathrm{CuPc}$ features are visible. By substraction of the CuPc spectrum, taken on a sample of CuPc on top of PEDOT:PSS, features of $\mathrm{C}_{60}$ can be displayed (dotted line).
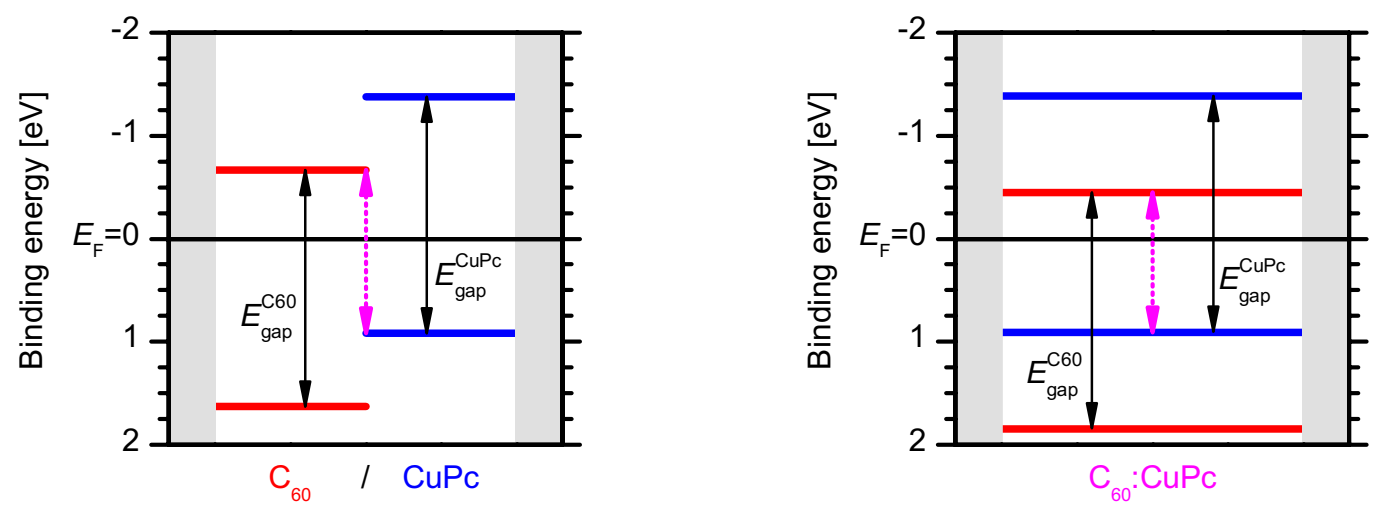

Figure 4. Schematic energy diagram for the bilayered (left side) and blended (right side) material system of CuPc and $\mathrm{C}_{60}$. The transport gap is $2.3 \mathrm{eV}$ for both neat materials, ${ }^{22,23}$ which is also assumed for the mixtures. ${ }^{15}$ The Fermi energy is related to the conductive substrate. The dashed arrow indicates the magnitude of the intermolecular HOMO-LUMO gap in both cases.

rising edge of the spectrum between $2 \mathrm{eV}$ and $3 \mathrm{eV}$. To display the $\mathrm{C}_{60}$ features, the spectrum for a neat $\mathrm{CuPc}$ film on PEDOT:PSS (not shown) was subtracted from the presented measurements. The resulting curves are shown as dotted lines and contain the features of $\mathrm{C}_{60} \cdot{ }^{15,20}$ The measured spectra are dominated by the $\mathrm{CuPc}$ features because the $\mathrm{C}_{60}$ molecules are clustering on top of the $\mathrm{CuPc}$ film with some uncovered area for the bilayered system (comparable to $\mathrm{C}_{60}$ on PEDOT:PSS ${ }^{21}$ ) and the low $\mathrm{C}_{60}$ content of about $25 \%$ for the blended system. Since the built-in voltage in the diodes described before and the intermolecular gap between $\mathrm{C}_{60}$ and $\mathrm{CuPc}$ on gold electrodes ${ }^{13,15}$ is independent on the mixing ratio the determined intermolecular gap should be comparable to the one in a 1:1 mixture analysed in solar cells below.

The comparison of the two systems shows no significant change of the energetic position of the CuPc HOMO, but a higher binding energy of the $\mathrm{C}_{60} \mathrm{HOMO}$ in the blended system. Concerning a transport gap of $2.3 \mathrm{eV}$ for both materials ${ }^{22,23}$ and assuming the same value in the blended film ${ }^{15}$ the energy diagram for the bilayered and the blended system is shown in figure 4 . The energy levels are determined from the low energy edge of the HOMO levels relative to the Fermi level. The transport gaps of both materials are represented by solid arrows. The bilayered system contains $\mathrm{CuPc}$ and $\mathrm{C}_{60}$ molecules in separated films while both molecule types are distributed 
over the whole film in the blended system. The intermolecular HOMO-LUMO gap is the important parameter for the open circuit voltage of photovoltaic cells. The values are $1.6 \mathrm{eV}$ and $1.35 \mathrm{eV}$ for the bilayered and the blended system, respectively. As an extension of previous results on gold electrodes ${ }^{15}$ the blended solar cells using PEDOT:PSS should thus also have a lower open circuit voltage than the bilayered solar cells. However, we note that the energy levels do not describe the real interface behaviour. While no band banding occurs at the organic/organic interface ${ }^{20}$ which is the relevant one for the photovoltaic cells, the electrode/organic interfaces are shown in figure 4 only schematically without interface dipoles or possible band bending.

\section{PHOTOVOLTAIC CELLS}

In the following the discussed results are compared to solar cell measurements. The current-voltage characteristics of both types of cells for different light intensities are shown in figure 5. The crossing point of the curves for different light intensities is the compensation voltage $V_{\mathrm{Comp}}{ }^{24}$ where the dark current and the photocurrents are equal. This compensation voltage is about $0.65 \mathrm{eV}$ for the bilayered system and about $0.4 \mathrm{eV}$ for the blended system. The difference of the compensation voltages for the two systems is thus equal to the difference between the intermolecular HOMO-LUMO gaps determined by UPS measurements. The open circuit voltages $\left(V_{\mathrm{OC}}\right)$ for
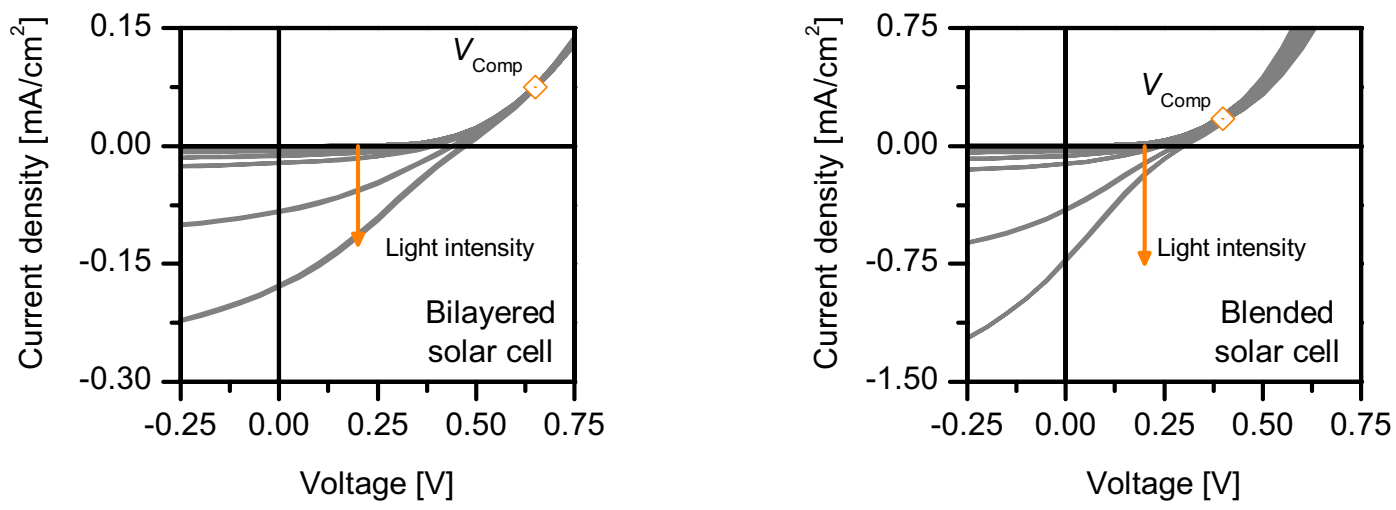

Figure 5. Current-voltage characteristics for bilayered and blended solar cells. The curves are shown for different light intensities. The compensation voltage $V_{\text {Comp }}$ is determined as the crossing point of all curves for different light intensities. ${ }^{24}$
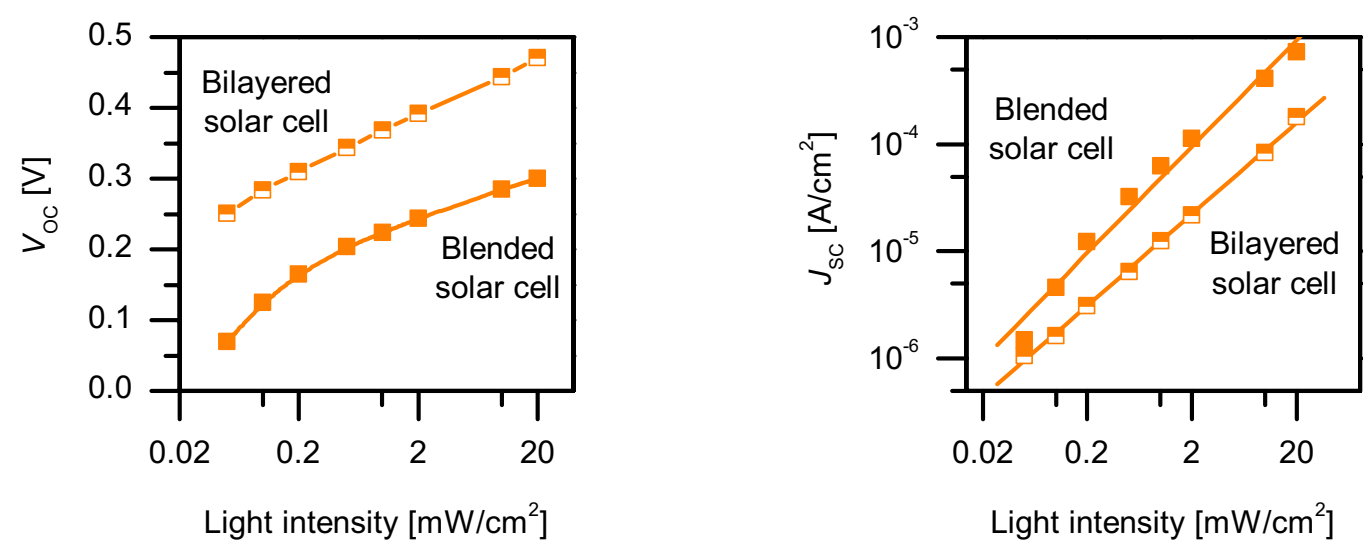

Figure 6. Open circuit voltage $\left(V_{\mathrm{OC}}\right)$ and short circuit current density $\left(J_{\mathrm{SC}}\right)$ for bilayered and blended solar cells as function of the white light intensity. The symbols are determined from the measurements shown in figure 5 . The lines are to guide the eyes. 

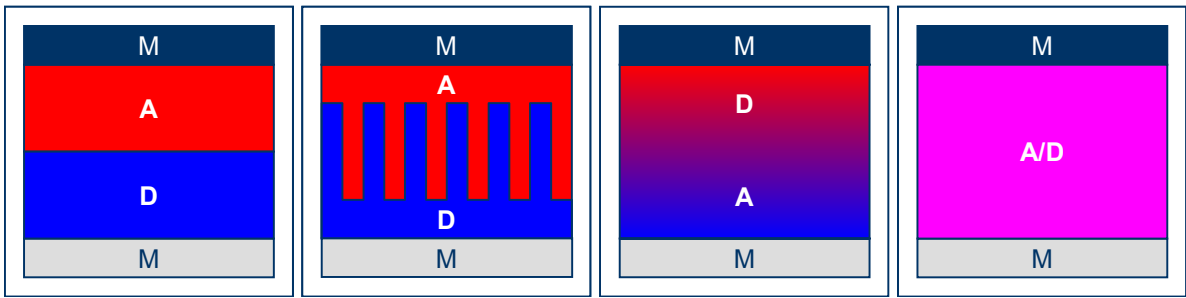

Figure 7. Different structures for solar cells. From left to right: planar heterojunction (bilayered structure), planar D/A layers combined with interdigitated interface, gradient heterojunction, bulk heterojunction (blended structure).

the different light intensities are collected in figure 6 on the left side. Over the whole range of the different light intensities the open circuit voltage in the bilayered cell is about $0.15 \mathrm{~V}$ higher than in the blended system. This effect was already observed in the $\mathrm{CuPc} / \mathrm{C}_{60}$ system at high illumination intensities, ${ }^{9}$ as well as for other material combinations using small molecules ${ }^{25}$ or polymers. ${ }^{26}$ The open circuit voltage is therefore related to the gap between the LUMO of the acceptor and the HOMO of the donor. ${ }^{27}$ Both the measured open circuit voltage and the compensation voltage are smaller for the blended system as expected from the UPS measurements. However, the open circuit voltage is significantly smaller than the intermolecular gap. This is related to further losses inside the organic photovoltaic cell. ${ }^{27}$ Nevertheless changes of the electronic levels of the donor and acceptor molecules are seen directly as a change of the open circuit voltage.

Figure 6 (right) compares the short circuit current density $\left(J_{\mathrm{SC}}\right)$ of both systems. The blended solar cell is providing the higher short circuit current. The reason is that by the mixing of the molecules a distributed donor/acceptor interface is formed inside the organic film. Thereby it is possible for nearly all excitons to reach the donor/acceptor interface to dissociate even for a short exciton diffusion length. It is remarkable that the short circuit currents for the blended solar cells are higher than for the bilayered solar cells even though the mobility in the blended system is by far lower than in the neat films described above and in the literature. ${ }^{9,12}$ The fill factor reaches $41 \%$ for the bilayered cell and $36 \%$ for the blended cell at a light intensity of about $0.5 \mathrm{~mW} / \mathrm{cm}^{2}$. Due to series resistances and recombination losses the power efficiency is rather low in our cells (at most $0.5 \%$ for the blended and maximal $0.3 \%$ for the bilayered solar cell).

\section{SUMMARY}

This contribution compares the charge carrier transport in neat and blended diodes as well as the electronic structure and the solar cell performance for bilayered (heterojunction) solar cells and blended (bulk-heterojunction) solar cells. The electronic structure shows a higher intermolecular gap resulting in a higher open circuit voltage for bilayered solar cells. Nevertheless, the blended solar cells reach higher short circuit currents based on the larger donor/acceptor interface in the blends. To combine the high open circuit voltage with high short circuit currents the investigation of other solar cell geometries might require. Figure 7 shows in addition to the blended and bilayered system a structure with interdigitated donor/acceptor films and with an active layer using a gradient from the neat donor to the neat acceptor material. A further possibility is doping of the material with the lower charge carrier mobility (here $\mathrm{CuPc}$ ) to increase the conductivity. ${ }^{28}$ These issues will be the subject of further ongoing studies.

\section{ACKNOWLEDGEMENT}

This work was supported by the Deutsche Forschungsgemeinschaft through Schwerpunktprogramm 1121 and Sonderforschungsbereich 484. The authors thank Jens Pflaum (University of Stuttgart) for purifying organic materials. Pierre Lorenz, Marcel Himmerlich, Stefan Krischok and Juergen A. Schaefer (Technical University of Ilmenau) have supported the photoelectron spectroscopy measurements. 


\section{REFERENCES}

[1] Sariciftci, N. S., Smilowitz, L., Heeger, A. J., and Wudl, F., "Photoinduced electron-transfer from a conducting polymer to buckminsterfullerene," Science 258, 1474-1476 (1992).

[2] Kraabel, B., McBranch, D., Sariciftci, N. S., Moses, D., and Heeger, A. J., "Ultrafast spectroscopic studies of photoinduced electron-transfer from semiconducting polymers to $\mathrm{C}_{60}$," Phys. Rev. B 50, 18543-18552 (1994).

[3] Tang, C. W., "Two-layer organic photovoltaic cell," Appl. Phys. Lett. 48, 183-185 (1986).

[4] Yu, G., Gao, J., Hummelen, J. C., Wudl, F., and Heeger, A. J., "Polymer photovoltaic cells - enhanced efficiencies via a network of internal donor-acceptor heterojunctions," Science 270, 1789-1791 (1995).

[5] Rostalski, J. and Meissner, D., "Monochromatic versus solar efficiencies of organic solar cells," Sol. Energ. Mat. Sol. C. 61, 87-95 (2000).

[6] Stübinger, T. and Brütting, W., "Exciton diffusion and optical interference in organic donor-acceptor photovoltaic cells," J. Appl. Phys. 90, 3632-3641 (2001).

[7] Peumans, P., Yakimov, A., and Forrest, S. R., "Small molecular weight organic thin-film photodetectors and solar cells," J. Appl. Phys. 93, 3693-3723 (2003).

[8] Heutz, S., Sullivan, P., Sanderson, B. M., Schultes, S. M., and Jones, T. S., "Influence of molecular architecture and intermixing on the photovoltaic, morphological and spectroscopic properties of $\mathrm{CuPc} \mathrm{C}_{60}$ heterojunctions," Sol. Ener. Mater. Sol. Cell. 83, 229-245 (2004).

[9] Xue, J. G., Rand, B. P., Uchida, S., and Forrest, S. R., "Mixed donor-acceptor molecular heterojunctions for photovoltaic applications. II. Device performance," J. Appl. Phys. 98, 124903 (2005).

[10] Rand, B. P., Xue, J. G., Uchida, S., and Forrest, S. R., "Mixed donor-acceptor molecular heterojunctions for photovoltaic applications. I. Material properties," J. Appl. Phys. 98, 124902 (2005).

[11] Meijer, E. J., De Leeuw, D. M., Setayesh, S., Van Veenendaal, E., Huisman, B. H., Blom, P. W. M., Hummelen, J. C., Scherf, U., and Klapwijk, T. M., "Solution-processed ambipolar organic field-effect transistors and inverters," Nature Mater. 2, 678-682 (2003).

[12] Opitz, A., Bronner, M., and Brütting, W., "Ambipolar charge carrier transport in mixed organic layers of phthalocyanine and fullerene," J. Appl. Phys. 101, 063709 (2007).

[13] Bronner, M., Opitz, A., and Brütting, W., "Ambipolar charge carrier transport in organic semiconductor blends of phthalocyanine and fullerene," phys. stat. sol. (a) 205, 549-563 (2008).

[14] Loi, M. A., Rost-Bietsch, C., Murgia, M., Karg, S., Rieß, W., and Muccini, M., "Tuning optoelectronic properties of ambipolar organic light- emitting transistors using a bulk-heterojunction approach," Adv. Func. Mater. 16, 41-47 (2006).

[15] Opitz, A., Bronner, M., Brütting, W., Himmerlich, M., Schaefer, J. A., and Krischok, S., "Electronic properties of organic semiconductor blends: ambipolar mixtures of phthalocyanine and fullerene," Appl. Phys. Lett. 90, 212112 (2007).

[16] Lozzi, L., Granato, V., Picozzi, S., Simeoni, M., La Rosa, S., Delly, B., and Santucci, S., "CuPc:C 60 blend film: A photoemission investigation," J. Vac. Sci. E Technol. A 24, 1668-1675 (2006).

[17] Brütting, W., Berleb, S., and Muckl, A. G., "Device physics of organic light-emitting diodes based on molecular materials," Organic Electronics 2, 1-36 (2001).

[18] Mott, N. and Gurney, R., [Electronic Processes in Ionic Crystals], Clarendon Press, Oxford (1940).

[19] Murgatroyd, P., "Theory of space-charge limited current enhanced by frenkel effect," J. Phys. D: Appl. Phys. 3, 151-156 (1970).

[20] Molodtsova, O. V. and Knupfer, M., "Electronic properties of the organic semiconductor interfaces $\mathrm{CuPc} / \mathrm{C}_{60}$ and $\mathrm{C}_{60} / \mathrm{CuPc}, "$ J. Appl. Phys. 99, 053704 (2006).

[21] Zhang, F. J., Vollmer, A., Zhang, J., Xu, Z., Rabe, J. P., and Koch, N., "Energy level alignment and morphology of interfaces between molecular and polymeric organic semiconductors," Org. Electron. 8, 606614 (2007).

[22] Hill, I., Kahn, A., Soos, Z., and Pascal, R., "Charge-separation energy in films of p-conjugated organic molecules," Chem. Phys. Lett. 327, 181-188 (2000).

[23] Lof, R. W., Vanveenendaal, M. A., Koopmans, B., Jonkman, H. T., and Sawatzky, G. A., "Band-gap, excitons, and coulomb interaction in solid $\mathrm{C}_{60}, "$ Phys. Rev. Lett. 68, 3924-3927 (1992). 
[24] Mihailetchi, V. D., Koster, L. J. A., Hummelen, J. C., and Blom, P. W. M., "Photocurrent generation in polymer-fullerene bulk heterojunctions," Phys. Rev. Lett. 93, 216601 (2004).

[25] Melzer, C., Krasnikov, V. V., and Hadziioannou, G., "Charge transport, injection, and photovoltaic phenomena in oligo(phenylenevinylene) based diodes," J. Polym. Sci. B 41, 2665-2673 (2003).

[26] Hayashi, Y., Yamada, I., Takagi, S., Takasu, A., Soga, T., and Jimbo, T., "Influence of structure and $\mathrm{C}_{60}$ composition on properties of blends and bilayers of organic donor-acceptor polymer/ $\mathrm{C}_{60}$ photovoltaic devices," Jpn. J. Appl. Phys. 144, 1296-1300 (2005).

[27] Brabec, C. J., Dyakonov, V., Parisi, J., and Sariciftci, N. S., eds., [Organic Photovoltaics], ch. Semiconductor Aspects of Organic Bulk Heterojunction Solar Cells by C.J Brabec, 159-248, Springer-Verlag Berlin Heidelberg New York (2003).

[28] Pfeiffer, M., Beyer, A., Plonnigs, B., Nollau, A., Fritz, T., Leo, K., Schlettwein, D., Hiller, S., and Wohrle, D., "Controlled p-doping of pigment layers by cosublimation: Basic mechanisms and implications for their use in organic photovoltaic cells," Sol. Ener. Mater. Sol. Cell. 63, 83-99 (2000). 